\title{
RhoC is essential in TGF- $\beta 1$ induced epithelial-mesenchymal transition in cervical cancer cells
}

\author{
XIAOQI HE, YING QIAN, HUILAN CAI, SHOUHUA YANG, JING CAI and ZEHUA WANG \\ Department of Obstetrics and Gynecology, Union Hospital, Tongji Medical College, \\ Huazhong University of Science and Technology, Wuhan, Hubei 430022, P.R. China
}

Received August 16, 2014; Accepted April 17, 2015

DOI: 10.3892/ol.2015.3287

\begin{abstract}
Epithelial-mesenchymal transition (EMT) is a critical process in the promotion of epithelial tumor progression and metastasis. The present study aimed to investigate the role of Ras homolog gene family, member C (RhoC) guanosine triphosphatase (GTPase) in transforming growth factor (TGF)- $\beta 1$ induced EMT. EMT occurred in human cervical carcinoma $\mathrm{SiHa}$ cells following TGF- $\beta 1$ stimulation for 4 days, as demonstrated by the appearance of mesenchymal morphology, reorganization of the actin cytoskeleton, reduced E-cadherin expression and increased Vimentin expression, which was associated with increased RhoC expression and activity. However, EMT was not observed in cells that were pretreated with RhoC siRNA prior to TGF- $\beta 1$ stimulation. Downregulation of RhoC 4 days following TGF- $\beta 1$ stimulation was not able to reverse the existing EMT. In addition, TGF- $\beta 1$ promoted the invasion of the control SiHa cells but not that of the cells in which RhoC was downregulated. In conclusion, RhoC expression is activated by TGF- $\beta 1$, and sufficient RhoC expression levels are essential for TGF- $\beta 1$-induced EMT.
\end{abstract}

\section{Introduction}

The expected five-year survival rate for early-stage cervical cancer is $91 \%$. However, the five-year survival rate for cervical cancer in an advanced stage is dramatically reduced to $12 \%$, despite advances in surgical and radiation treatments (1). Thus, a deeper understanding of the mechanism underlying the progression of cervical cancer is essential for improving the prognosis of advanced tumors. Epithelial-mesenchymal transition (EMT) induced by transforming growth factor- $\beta$ (TGF- $\beta$ )

Correspondence to: Professor Zehua Wang, Department of Obstetrics and Gynecology, Union Hospital, Tongji Medical College, Huazhong University of Science and Technology, 1,277 Jiefang Avenue, Wuhan, Hubei 430022, P.R. China E-mail: zehuawang@163.net

Key words: epithelial-mesenchymal transition, Ras homolog gene family, member C guanosine triphosphatase, RNA interference, invasion, cervical cancer is well-established as a critical mechanism of tumor progression (2). Epithelial cells transdifferentiate into fibroblast-like cells, which results in a lack of adhesion and actin cytoskeleton reorganization, enhancing the migratory and invasive properties of cells (3). In addition, EMT is characterized by alterations in the expression of a series of biomarkers, in particular, reduced E-cadherin and increased Vimentin expression levels. Ras homolog gene family, member $\mathrm{C}$ guanosine triphosphatase (RhoC GTPase) is an important member of the Rho-GTPase family, which regulates actin cytoskeleton reorganization during cellular motility, and actively participates in the EMT process (4-6). However, the role of RhoC in the process of EMT has remained to be elucidated. Since the majority of cervical cancer cases are squamous cell carcinomas and associated with high risk HPV infection, the present study used the SiHa cell line which is HPV16 positive for the investigation. In the present study, a cellular model of TGF- $\beta 1$-induced EMT was established in the SiHa human cervical cancer cell line and the alterations in RhoC activity and expression were investigated, in addition to the effect of RhoC inhibition on EMT.

\section{Materials and methods}

Cell culture. SiHa cells derived from human cervical squamous carcinoma were obtained from the American Type Culture Collection (Manassas, VA, USA). The cells were cultured in Dulbecco's modified Eagle's medium (Gibco Life Technologies, Carlsbad, CA, USA ) containing $10 \%$ fetal calf serum (Gibco Life Technologies) and incubated at $37^{\circ} \mathrm{C}$ in $5 \% \mathrm{CO}_{2}$. TGF- $\beta 1$ (PeproTech, Inc., Rocky Hill, NJ, USA) was added to the culture medium at $10 \mathrm{ng} / \mathrm{ml}$, and the culture medium containing TGF- $\beta 1$ was changed every other day. The cells were cultured for 7 days and observed daily; cells cultured without TGF- $\beta 1$ were used as control (7).

Immunofluorescence microscopy. The cells were cultured on coverslips until they reached $60 \%$ confluence and were subsequently fixed in $4 \%$ paraformaldehyde (Sigma-Aldrich, Santa Clara, CA, USA) for $15 \mathrm{~min}$, permeabilized with $0.5 \%$ Triton $\mathrm{X}-100 /$ phosphate-buffered saline (Sigma-Aldrich) for $10 \mathrm{~min}$ and then blocked with confining liquid (0.1\% Triton X-100/PBS, $2 \%$ BSA, $0.1 \%$ Sodum Azide; Sigma-Aldrich) for $10 \mathrm{~min}$. The cells were then stained with Alexa FITC-phalloidin (dilution 1:20, Alexion Pharmaceuticals, Lausen, Switzerland) for 
10 min and observed under a FV500 laser confocal microscope (Olympus Corporation, Tokyo, Japan). The cells were incubated with rabbit polyclonal anti-E-cadherin antibody (dilution 1:50; Boster Biologics, Pleasanton, CA, USA) or mouse monoclonal anti-Vimentin (dilution 1:20; Thermo Fisher Scientific, Inc., Waltham, MA, USA) antibody at $4^{\circ} \mathrm{C}$ overnight and stained with specific FITC-conjugated secondary antibodies (mouse antirabbit IgG-FITC, dilution 1:300, Santa Cruz Biotechnology, Dallas, TX, USA, sc-2359; or goat anti-mouse IgM-FITC, dilution 1:400, Santa Cruz biotechnology, Dallas, TX, USA, sc-2010) for $60 \mathrm{~min}$ at room temperature and viewed under a IX71 fluorescence microscope (Olympus Corporation).

Western blot analysis. The cells were lysed in a lysis buffer and the proteins were separated on 12\% SDS-polyacrylamide gels and transferred to nitrocellulose membranes (SigmaAldrich). The membrane was blocked with a 5\% milk solution in TBST (Tris-HCl-buffered saline supplemented with $0.5 \%$ Tween-20; Sigma-Aldrich), and incubated with a primary antibodies (goat polyclonal IgG anti-RhoC, rabbit polyclonal $\operatorname{IgG}$ anti-E cadherin and goat polyclonal IgG anti- $\beta$ actin, 1:200, Santa Cruz Biotechnology, sc-12116, sc-7870 and sc-1616; mouse monoclonal IgM anti-vimentin, 1:200, Themo Fisher Scienfic, Walthan, MA, USA, MA3-745) in 5\% milk/TBST overnight, followed by incubation with the corresponding horseradish peroxidase-linked secondary antibodies (1:5000, Santa Cruz Biotechnology; sc-2922, sc-2357 and sc-2005). Following extensive washing, the signals were detected using an enhanced chemiluminscence-detecting reagent $(\bullet \mathrm{GE}$ Healthcare Life Sciences, Chalfont, UK). For each western blotting result, the experiment was performed $\geq 3$ times, and representative images are presented in the results.

RhoC activity assay. RhoC activity was assessed using the Rho-binding domain of Rhotekin (RBD; Upstate Biotechnology, Temecula, CA, USA) according to the manufacturer's instructions. Briefly, the cells were lysed in radioimmunoprecipitation assay buffer $[50 \mathrm{mM}$ Tris $(\mathrm{pH} 7.2), 1 \%$ Triton X-100, $0.5 \%$ sodium deoxycholate, $0.1 \% \mathrm{SDS}, 500 \mathrm{mM} \mathrm{NaCl}, 10 \mu \mathrm{g} / \mathrm{ml}$ each of leupeptin and aprotinin and $1 \mathrm{mM}$ phenylmethanesulfonylfluoride (PMSF); Sigma-Aldrich]. The cell lysates were clarified by centrifugation at $13,000 \mathrm{x}$ g for $10 \mathrm{~min}$ at $4^{\circ} \mathrm{C}$ and were subsequently incubated for 40 min with Rhotekin Rho Binding Domain-agarose. The agarose beads were washed 3 times with washing buffer comprised of Tris buffer containing $1 \%$ Triton X-100, $150 \mathrm{mM} \mathrm{NaCl}, 10 \mathrm{mM} \mathrm{MgCl} 2$, $10 \mu \mathrm{g} / \mathrm{ml}$ each of leupeptin and aprotinin and $0.1 \mathrm{mM}$ PMSF. The Rho that bound to the beads was the GTP-bound form of the Rho protein and was detected by western blotting using RhoC antibodies.

In vitro cell invasion assay. A modified cell migration assay was performed using a Transwell apparatus $(8-\mu \mathrm{m}$ pore; Corning Life Sciences, Cambridge, MA, USA) as previously described (8). Briefly, the upper surface of the Transwell chambers were pre-coated overnight with Matrigel (BD Biosciences, Franklin Lakes, NJ, USA) diluted 1:4 in serum-free medium. The cell suspension $\left(2.5 \times 10^{5}\right.$ cells in $100 \mu 1$ serum-free medium) was transferred to a polycarbonate filter (pore size, $8 \mu \mathrm{m}$ ) in the upper compartment of the apparatus, and $400 \mu \mathrm{l}$ complete culture medium was placed in the lower compartment. The apparatus was then placed in a humidified incubator containing $5 \% \mathrm{CO}_{2}$ for $24 \mathrm{~h}$ at $37^{\circ} \mathrm{C}$. Cells that migrated through the membrane were exposed to hematoxylin and eosin staining. The number of cells penetrating the membrane was determined by evaluating 5 independent high-power fields in triplicate at x400 magnification, using a under a CX41-72C02 microscope (Olympus Corporation).

RhoC short interfering (si)RNA transfection. A specific siRNA directed against human RhoC messenger RNA was designed and synthesized by Shanghai GenePharma Co., Ltd. (Shanghai, China). The sequences selected for anti-RhoC siRNA were: Sense, 5'-ACUGUCUUGAGAACUAUATT-3' and antisense, 5'-UAUAGUUCUCAAAGACAGUAG-3'. The siRNA was introduced into SiHa cells by RNAi-Mate transfection reagents (Shanghai GenePharma Co., Ltd.), according to the manufacturer's instructions. The cells were cultured in 6 -well plates in $2 \mathrm{ml}$ serum-enriched medium. When $80 \%$ confluence was reached, $5 \mu \mathrm{g}$ siRNA and $15 \mu \mathrm{l}$ RNAi-Mate mixture was added to the cell cultures and incubation was continued for $48 \mathrm{~h}$.

Statistical analysis. Results are expressed as the mean \pm standard deviation. A Student's t-test was used to perform comparisons using SPSS software, version 12.1 (SPSS, Inc., Chicago, IL, USA). P $<0.05$ was considered to indicate a statistically significant difference.

\section{Results}

TGF- $\beta 1$ induces EMT in SiHa cells. Following 2 days of $10 \mathrm{ng} / \mathrm{ml}$ TGF- $\beta 1$ treatment, the morphology of the SiHa cells began to change. The cells were enlarged and lengthened, and exhibited a spindle-like shape. The cells demonstrated a distinct spindle-like shape 4 days later. Seven days later, the morphological alterations were marked: The cells were arranged in a paliform shape, and the length was increased by $\sim 7$ fold compared with that of the normal control cells cultured without TGF- $\beta 1$ (Fig. 1A).

In addition, TGF- $\beta 1$ stimulation resulted in reorganization of the actin cytoskeleton, from a regular arrangement of circumferential ring-like structures into actin stress fibers, which were aggregated, broken and arranged in a disordered manner. Immunofluorescent staining demonstrated an E-cadherin fluorescent signal in the SiHa cell membrane, which was reduced or absent following TGF- $\beta 1$ treatment. Simultaneously, Vimentin was expressed de novo, which manifested as the aggregation of radiating filaments around the peripheral region of the nuclei (Fig. 1B). The alterations in expression levels of E-cadherin and Vimentin were confirmed by western blotting (Fig. 1C). The results of the present study provided morphological and molecular evidence that EMT occurred in the SiHa cells subjected to TGF- $\beta 1$ treatment.

RhoC is essential for TGF- $\beta 1$-induced EMT. Western blot analysis demonstrated that the RhoC and RhoC-GTP levels were increased following 4 days of $10 \mathrm{ng} / \mathrm{ml}$ TGF- $\beta 1$ treatment (Fig. 2A), indicating that TGF- $\beta 1$ may increase RhoC protein expression and activity. 

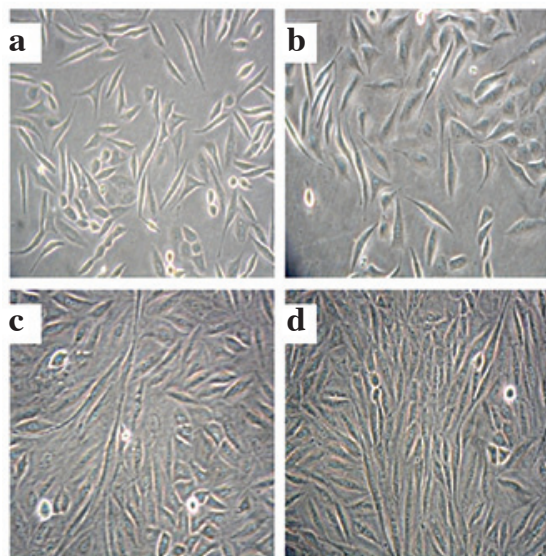

$\mathbf{C}$

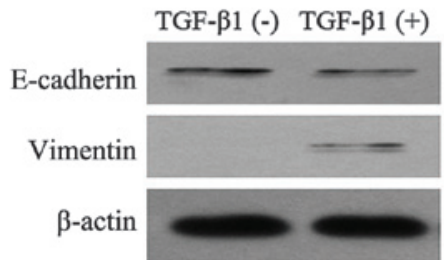

B
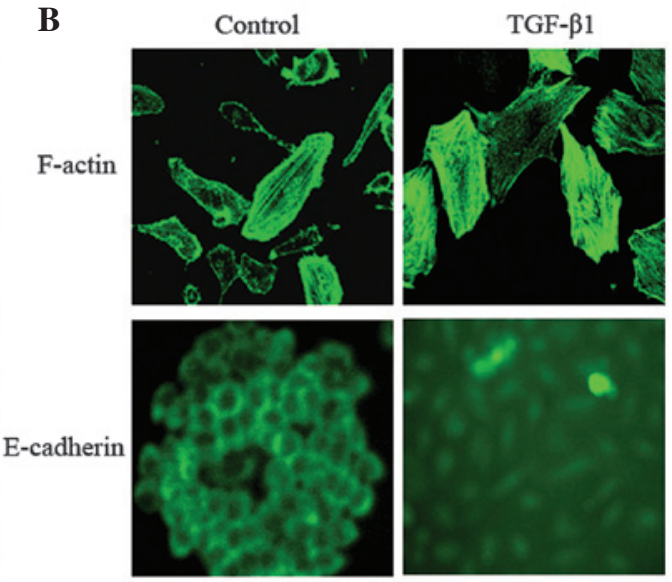

Vimentin

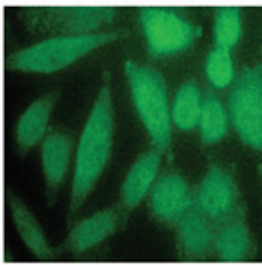

Figure 1. TGF- $\beta 1$ induces epithelial-mesenchymal transition in SiHa cells. (A) Morphological changes of SiHa cells following (a) 0 , (b) 2 , (c) 4 and (d) 7 days of TGF- $\beta 1$ treatment. (B) Immunofluorescent staining of F-actin, E-cadherin and Vimentin in SiHa cells following 4 days of TGF- $\beta 1$ treatment. (C) Western blot analysis of E-cadherin, Vimentin and $\beta$-actin expression with or without 4 days of TGF- $\beta 1$ treatment. E-cadherin expression was reduced and Vimentin expression was increased. TGF- $\beta 1$, transforming growth factor- $\beta 1$.

A

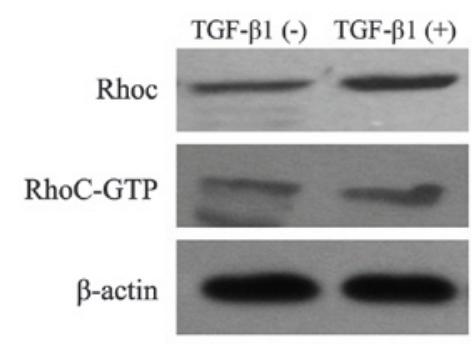

C

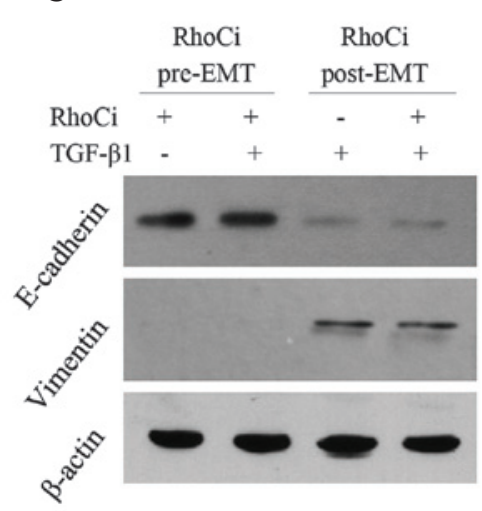

B
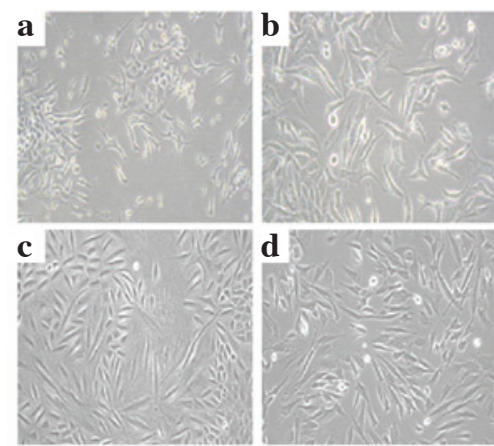

D
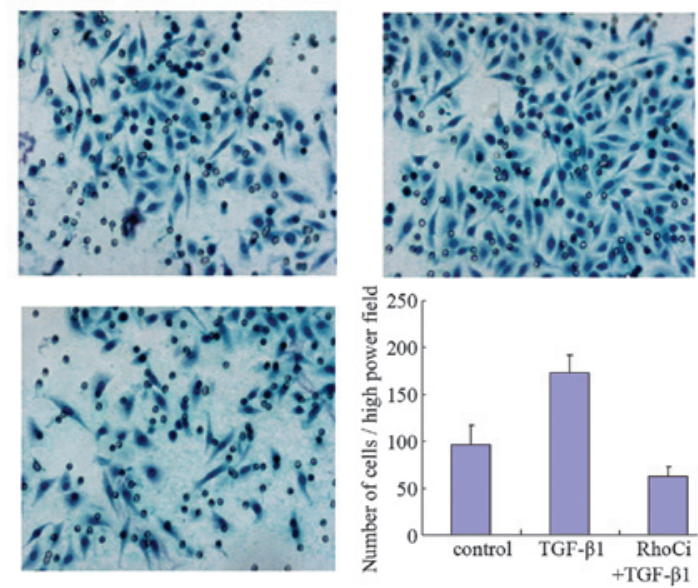

Figure 2. RhoC is essential for TGF- $\beta 1$-induced EMT in SiHa cells. (A) Western blot analysis indicated that the expression of RhoC protein was increased following TGF- $\beta 1$ stimulation and resulted in enhanced activity. (B) SiHa cells were pretreated with RhoC siRNA: (a) Cells remained epithelial phenotype following stimulation with $10 \mathrm{ng} / \mathrm{ml}$ TGF $\beta 1$ for 4 days. SiHa cells were treated with (b) $10 \mathrm{ng} / \mathrm{ml}$ TGF $\beta 1$ to induce EMT; (c) then RhoC siRNA transfection; (d) no morphological alterations were observed following 4 days. (C) Western blot analysis demonstrated that downregulation of RhoC prevented the TGF- $\beta 1$-induced effects on the expression of E-cadherin and Vimentin, although it did not reverse existing EMT induced by TGF- $\beta 1$. (D) The invasive activity of cells was assayed in a Matrigel coated Transwell. TGF- $\beta 1$ promoted control SiHa cell penetration of the Matrigel and membrane; this was not observed in cells pretreated with RhoC short interfering RNA undergoing TGF- $\beta 1$ treatment. The cells that penetrated the membrane were counted and the results are presented as the mean \pm standard deviation in the histogram. EMT, epithelial-mesenchymal-transition; TGF- $\beta 1$, transforming growth factor- $\beta 1$; RhoC, Ras homolog gene family, member $\mathrm{C}$. 
To investigate the role of RhoC in EMT in cervical cancer cells, RhoC was silenced in SiHa cells by siRNA transfection prior to TGF- $\beta 1$ treatment or four days following TGF- $\beta 1$ treatment. The cells in which $\mathrm{RhoC}$ was downregulated did not exhibit a mesenchymal morphology (Fig. 2B) or alterations in the expression of EMT markers, E-cadherin and Vimentin (Fig. 2C), following TGF- $\beta 1$ treatment. This result indicated that $\mathrm{RhoC}$ is required for the process of EMT. However, silencing of RhoC following pretreatment with TGF- $\beta 1$ did not alter the incidence of EMT (Figs. 2B and C). In addition, following TGF- $\beta 1$ stimulation, the invasiveness of $\mathrm{SiHa}$ cells was elevated $\sim 2$-fold $(\mathrm{P}<0.01)$, while this phenomenon was not observed in cells in which $\mathrm{RhoC}$ was silenced $(\mathrm{P}>0.05$; Fig. 2D). These results indicate that inhibition of RhoC expression may block the process of TGF- $\beta 1$-induced EMT, however it is insufficient to reverse EMT.

\section{Discussion}

EMT has been observed in several epithelial tumors, including oral squamous cell carcinoma (9), breast cancer (10), endometrial cancer (11) and pancreatic carcinoma (12). TGF- $\beta 1$ has been demonstrated to be able to induce EMT, and is important in tumor development (13-16).

In the present study, SiHa cells underwent an EMT process following TGF- $\beta 1$ treatment: The cells were clearly enlarged and spindle-shaped and E-Cadherin levels were significantly reduced, while Vimentin levels were increased. A reduction in the expression of E-cadherin initiates a series of reactions which finally result in breakdown of the intercellular tight junctions between cells, allowing cells to gain movement capability (4). A previous study demonstrated that the expression level of E-cadherin was reduced in EMT (17).

TGF- $\beta 1$-induced cytoskeletal remodeling indicates that RhoC, which serves an important function in regulating stress fiber formation, may participate in the TGF- $\beta 1$-induced EMT process. In order to examine this hypothesis, RhoC expression was analyzed in TGF- $\beta 1$-treated cells. Following TGF- $\beta 1$ stimulation, the RhoC protein expression levels in SiHa cells were significantly increased compared with those of the blank control group, and RhoC GTPase activity was also significantly increased. Similarly, Cho and Yoo (4) demonstrated that TGF- $\beta 1$ serves a dual role in inducing Rho activity, instantly and temporarily increasing activity, followed by a slower increasing stage. A similar dual activation phenomenon of Rho was also observed in prostate adenocarcinoma (18). The mechanism of this dual activation remains to be elucidated. However, in the present study, due to the various observation time-points that were selected, this phenomenon was not observed.

The present study also demonstrated that when the RhoC expression levels increased, the invasive capability of the SiHa cells was also increased. When the RhoC expression levels were pre-inhibited, the invasive capability of the SiHa cells was significantly reduced, even with continuous TGF- $\beta 1$ stimulation. These data indicated that the enhancement of TGF- $\beta 1$-induced cellular invasive capability is dependent on upregulation of RhoC expression. Simpson et al (19) reported that when RhoA was inhibited, RhoC promoted lysophosphatidic acid-induced MCF-7 breast cancer cell invasive capability. The authors also observed that inhibition of RhoA gene expression increased the invasive capacity of the MCF-7 cells. Analogous effects were observed in colon cancer (6). Taken together, the findings of previous studies and the present study, indicate that knockdown of the RhoC gene may inhibit the TGF- $\beta 1$ induced invasiveness of $\mathrm{SiHa}$ cells.

In addition, the association between RhoC and EMT was further investigated. A RhoC-specific siRNA was transfected into $\mathrm{SiHa}$ cells, prior to TGF- $\beta 1$ treatment and following EMT occurrence, respectively. Inhibition of RhoC expression blocked the EMT process, however, if EMT had already occurred in the cells, then silencing of RhoC using the siRNA was not sufficient to reverse the EMT process and the associated morphological changes. Cho and Yoo (4) demonstrated that the Rho-Rock inhibitor, Y27632, was able to completely block TGF- $\beta 1$-induced EMT. Another previous study demonstrated that a RhoA-specific inhibitor did not delay or block TGF- $\beta 1$-induced EMT in SiHa cells (7). Therefore, a comparison of RhoA and RhoC expression levels and the alterations in their activity may be critical in further understanding the TGF- $\beta 1$-induced EMT process.

EMT is a complex biological process, involving multiple signaling pathways associated with cancer formation and development. In addition, these intracellular pathways may cross-talk with one another (20). A number of proteins that are involved in the EMT process are also tumor malignant process marker proteins (21). Therefore, EMT may be a critical process in recognizing the relevant molecular expression patterns in invasive disease, and may also represent a potential target in tumor therapy $(21,22)$. In conclusion, targeted inhibition of RhoC activity may be potentially useful in the treatment of cancer.

\section{Acknowledgements}

The present study was supported by the National Natural Science Foundation of China (nos. 81072134 and 81202036).

\section{References}

1. Siegel R, Ma J, Zou Z and Jemal A: Cancer statistics, 2014. CA Cancer J Clin 64: 9-29, 2014.

2. Rees JR, Onwuegbusi BA, Save VE, et al: In vivo and in vitro evidence for transforming growth factor-betal-mediated epithelial to mesenchymal transition in esophageal adenocarcinoma. Cancer Res 66: 9583-9590, 2006.

3. Thiery JP: Epithelial-mesenchymal transitions in tumour progression. Nat Rev Cancer 2: 442-454, 2002.

4. Cho HJ and Yoo J: Rho activation is required for transforming growth factor- $\beta$-induced epithelial-mesenchymal transition in lens epithelial cells. Cell Biol Int 31: 1225-1230, 2007.

5. Mukai M, Endo H, Iwasaki T, et al: RhoC is essential for TGF-beta1-induced invasive capacity of rat ascites hepatoma cells. Biochem Biophys Res Commun 346: 74-82, 2006.

6. Bellovin DI, Simpson KJ, Danilov T, et al: Reciprocal regulation of RhoA and $\mathrm{RhoC}$ characterizes the EMT and identifies RhoC as a prognostic marker of colon carcinoma. Oncogene 25: 6959-6967, 2006.

7. Yi JY, Hur KC, Lee E, et al: TGFbeta1-mediated epithelial to mesenchymal transition is accompanied by invasion in the SiHa cell line. Eur J Cell Biol 81: 457-468, 2002.

8. Lu SD: Current Protocols for Molecular Biology. Publish House of Advanced Education, Beijing, pp381-385, 1993.

9. Chaw SY, Majeed AA, Dalley AJ, et al: Epithelial to mesenchymal transition (EMT) biomarkers - E-cadherin, beta-catenin, APC and Vimentin - in oral squamous cell carcinogenesis and transformation. Oral Oncol 48: 997-1006, 2012. 
10. Wang Y and Zhou BP: Epithelial-mesenchymal transition - a hallmark of breast cancer metastasis. Cancer Hallm 1: 38-49, 2013.

11. Mirantes C,Espinosa I,Ferrer I, Dolcet X,Prat J and Matias-Guiu X: Epithelial-to-mesenchymal transition and stem cells in endometrial cancer. Hum Pathol 44: 1973-1981, 2013.

12. Karamitopoulou E: Role of epithelial-mesenchymal transition in pancreatic ductal adenocarcinoma: Is tumor budding the missing link? Front Oncol 3: 221, 2013.

13. Wendt MK, Smith JA and Schiemann WP: Transforming growth factor- $\beta$-induced epithelial-mesenchymal transition facilitates epidermal growth factor-dependent breast cancer progression. Oncogene 29: 6485-6498, 2010

14. Shirakihara T, Horiguchi K, Miyazawa K, et al: TGF- $\beta$ regulates isoform switching of FGF receptors and epithelial-mesenchymal transition. EMBO J 30: 783-795, 2011.

15. Thiery JP, Acloque H, Huang RY and Nieto MA: Epithelial-mesenchymal transitions in development and disease. Cell 139: 871-890, 2009.

16. Heldin $\mathrm{CH}$, Vanlandewijck $\mathrm{M}$ and Moustakas A: Regulation of EMT by TGF $\beta$ in cancer. FEBS Lett 586: 1959-1970, 2012.
17. Reinacher-Schick A, Baldus SE, Romdhana B, et al: Loss of Smad4 correlates with loss of the invasion suppressor E-cadherin in advanced colorectal carcinomas. J Pathol 202: 412-420, 2004

18. Edlund S, Landström M, Heldin $\mathrm{CH}$ and Aspenström $\mathrm{P}$ : Transforming growth factor-beta-induced mobilization of actin cytoskeleton requires signaling by small GTPases Cdc42 and RhoA. Mol Biol Cell 13: 902-914, 2002.

19. Simpson KJ, Dugan AS and Mercurio AM: Functional analysis of the contribution of RhoA and RhoC GTPases to invasive breast carcinoma. Cancer Res 64: 8694-8701, 2004.

20. Thiery JP and Sleeman JP: Complex networks orchestrate epithelial-mesenchymal transitions. Nat Rev Mol Cell Biol 7: 131-142, 2006.

21. Spaderna S, Schmalhofer O, Hlubek F, et al: A transient, EMT-linked loss of basement membranes indicates metastasis and poor survival in colorectal cancer. Gastroenterology 131: 830-840, 2006.

22. Guarino M: Epithelial-mesenchymal transition and tumour invasion. Int J Biochem Cell Biol 39: 2153-2160, 2007. 